\title{
Slum-Tourismus und soziale
}

Stadtführungen

\section{Einblicke in soziale Brennpunkte und die "Soziale Frage"}

\section{UDO WILKEN}

Prof. Dr. phil. Udo Wilken, Diplompädagoge, Behindertenpädagoge und Pastor a. D., lehrt an der Fakultät Soziale Arbeit und Gesundheit der Hochschule für angewandte Wissenschaft und Kunst in Hildesheim. Aktuelle Forschungsschwerpunkte liegen im Bereich der Freizeit-KulturPädagogik und des barrierefreien Tourismus. Von 1992 bis 2006 war er Mitglied des Vorstandes der Deutschen Gesellschaft für Soziale Arbeit.

udo.wilken@gmx.de

\author{
Das Fremde in der Fremde und das Fremde im eigenen \\ Umkreis übte schon immer eine Faszination auf \\ Menschen aus. Verantwortungsvolle Exkursionen \\ in andere Länder wollen dieses Interesse für eine \\ Sensibilisierung der gesellschaftlichen Verhältnisse \\ ebenso nutzen wie neue Initiativen, die soziale \\ Stadtführungen in Deutschland zu Brennpunkten \\ von Armut und Migration anbieten.
}

Mit "Slum-Tourismus « wird die Begehung insbesondere von städtischen Armenvierteln in den touristischen Destinationen weltweit bezeichnet.

Bereits im 19. Jahrhundert verbreiteten sich entsprechende Touren durch die Elendsviertel der Industriestädte in Europa, als wohlsituierte Bürgerinnen und Bürger der Oberschicht im viktorianischen England mit solcher Art "Sightseeing « begannen. Später erfolgten vergleichbare Exkursionen in den USA durch die überbevölkerten Ghettos der Einwandererquartiere (Frenzel u.a. 2012, 2).

Mit zunehmender Globalisierung des Reisens führt der mittlerweile boomende Slum-Tourismus (Rolfes u. a. 2009, 11) vor allem in die Ghettos, Favelas und Townships der Megastädte in den Entwicklungs- und Schwellenländer, die für die Zugezogenen zur Armutsfalle, aber auch zur Entwicklungschance werden können.

Slums werden insbesondere im 19. Jahrhundert nicht nur als Orte krasser Armut und Not erlebt, sondern zugleich als Viertel der Verwahrlosung, der Gewalt und Kriminalität sowie erregender Lüsternheit. Von den abenteuerlichen Unternehmungen dorthin und der damit einhergehenden faszinierenden Konfrontation mit Armut, Elend und Not versprach man sich von Anfang an nicht allein einen Zeitvertreib mit Sensationslust und Nervenkitzel, son- dern ließ sich durchaus auch in Hinblick auf die mit Industrialisierung und Pauperisierung einhergehende zeittypische "Soziale Frage « empathisch berühren (Wendt 1990, 41, 125, 148).

Bezeichnet wurden sowohl die eher sozial-touristischen wie auch die von philanthropischen Sozialreformern unternommenen Exkursionen in Anlehnung an das Wort Armenviertel (engl. slum) mit dem Begriff »slumming «.

Um 1900 verwendeten zudem soziale Organisationen erstmals und gezielt Lichtbildserien und Kurzfilme, als damals neu entwickelte Medien, um »das Thema Armut ins Licht der Öffentlichkeit zu rücken. In Vortragssälen, Sonntagsschulen, Varieté-Theatern, Kinos und in der Kirche erlebten die Zuschauer Liveaufführungen ... bestehend aus Lichtbildern, Filmen, Musik, Gesang und Rezitation «, mit denen Armut, Not und Elend der Slums in Szene gesetzt wurden (Loiperdinger und Vogl-Bienek 2013, 10). Das Ziel bestand darin, die "Soziale Frage « im Bewusstsein der Menschen zu verankern, angemessene Hilfs- und Unterstützungsangebote zu erschließen und durch abschreckende Beispiele der medial inszenierten Unmoral, sozial-erzieherisch zu wirken und verantwortungsbewusstes Verhalten zu fördern (vgl. Screening the Poor 18881914. DVD-Edition 2011). 


\section{Slum-Tourismus als globale sozial-ethische Herausforderung}

Heutzutage begeben sich Touristen aus unterschiedlichen Gründen in Slums und Elendsviertel in unterentwickelten wie in entwickelten Ländern, aber auch zu »Schau-Plätzen « beklemmender sozialhistorischer Ereignisse in ihren weltweiten Urlaubsdestinationen.

$\mathrm{Ob}$ auf eigene Faust oder als geführte Tour, das Interesse reicht von der Besichtigung überbevölkerter prekärer Wohn- und Ansiedlungsgebiete mit Wellblechhütten-, traditionellen Höhlenbehausungen und auf Müllkippen schuftenden Kindern, über die Begehung von Drogenvierteln in Städten wie Amsterdam oder Kopenhagens Christiania - inklusive der dubiosen Konfrontation mit den Rotlicht-Milieus auf fünf Kontinenten (vgl. TourismWatch 2014a), bis hin zu touristischen Events, die sich auf den Spuren von Jack the Ripper und Sherlock Holmes bewegen und deren gruselige Endstation das Schafott im Tower of London bildet. Selbst bei einer New-York-Reise wird immer öfter jenseits von Glamour, Wallstreet und Börse als Kontrastprogramm der Besuch eines Sozialprojektes mit Kleiderkammer, sozialem Mittagstisch und Health-Care-Angeboten offeriert (vgl. Food Bank for New Yorkwww.foodbanknyc.org/; Church of the Holy Apostles - www.taz.de/!28394/).

Welche Motive und Einstellungen bei slum-touristischen Begehungen mitschwingen mögen, sei es neben einem be- wussten sozial-politischen Interesse auf der Grundlage spezifischer Vorkenntnisse, eine eher diffuse touristische Neugier nach sozial-kulturellen Authentizitätserlebnissen auf der Basis eines grundierten Gefühls mitmenschlicher Empathie und Solidarität mit Slum-Bewohnern, das sich mit der "Suche nach Alltagsdistanz, Differenzerfahrung und nach dem ganz Anderen « verbindet (M. Steinbrink, zitiert nach Gutekunst 2013), - beide, Organisatoren wie Teilnehmer solcher Exkursionen, benötigen angesichts der paradoxen Situation von Reich und Arm, sowohl bei der Begegnung mit der lokalen und regionalen Sozialgeschichte wie auch mit der von Armut und Elend bestimmten aktuellen Alltags- und Lebenswelt, in die die Touristen kurzzeitig eintauchen, ein soziales Fingerspitzengefühl, das insbesondere bei der unmittelbaren Begegnung mit den dort lebenden Menschen gefordert ist. Denn solche Unternehmungen sollten aus tourismus-ethischer Sicht nicht zu einer respektlos-entwürdigenden, sozial-voyeuristischen Touristenattraktion verkommen, bei der lediglich die Bereisten »begafft « und ihr Elend bigott konsumiert werden, sie selbst aber von diesen Touren weder direkt (in ökonomischer) noch indirekt (in unmittelbar erlebter wertschätzender Hinsicht) profitieren.

Angesichts eines mitunter idealisierten ambivalenten Bildes vom armen, obdachlosen, aber vermeintlich glücklichen Clochard unter der Seine-Brücke in Paris, bedarf es zumal im Blick auf den globalen

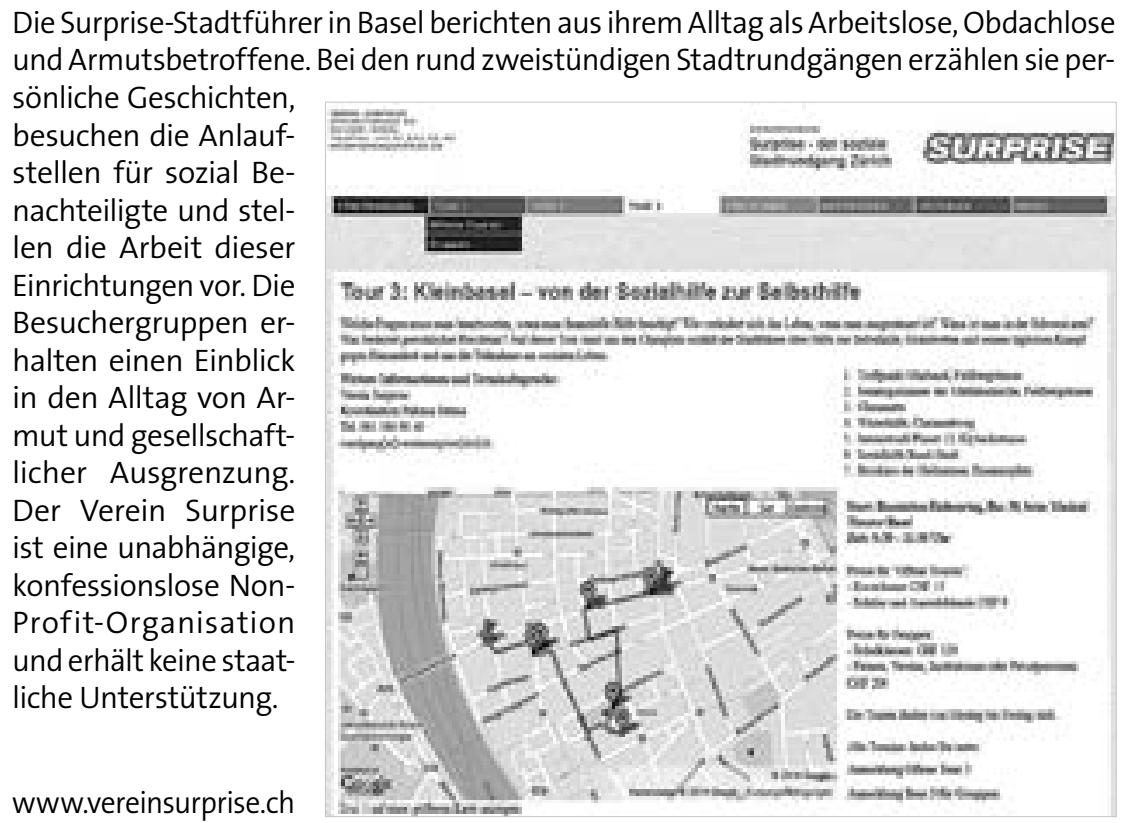

Slum-Tourismus durchaus sozialstruktureller Hintergrundinformationen bei den Touristen (vgl. TourismWatch 2014b) sowie einer interkulturellen Vermittlungskompetenz seitens der Tourveranstalter, gleich ob sie nun professionell-marktlich oder informell als Social-Profit-Organisationen auftreten.

Dadurch könnte im Blick auf die "Soziale Frage « ein realistischer sozialkultureller Perspektivenwechsel möglich werden, der nicht romantisch verklärt, sondern differenziert und sich gegebenenfalls nachhaltig förderlich zu engagieren weiß (vgl. Frenzel 2013).

Es ist deshalb wünschenswert, dass in Ergänzung zu den auch medial verbreiteten positiven wie auch negativen Stereotypien (vgl. die Internet-Kontroversen zum Kinofilm "Slumdog Millionaire« aus dem Jahr 2008), die Teilnehmer von Slum-Touren, wenn auch nur partiell, so doch exemplarisch, mit der komplexen, disparaten lebensweltlichen Alltagsrealität der Menschen dort konfrontiert werden (vgl. Rolfes u.a. 200951 ff.).

Vielleicht wächst dadurch die Chance, dass sich zusammen mit einem kritisch-reflektierenden sozial-kulturellen Einblick bei den Slum-Touristen mitmenschliche Sympathie und Respekt vor der Überlebensleistung der unter extrem desolaten Verhältnissen lebenden Menschen entwickeln und vertiefen kann.

\section{Touristische Begegnungen mit Elend und Migration in Deutschland}

In Deutschland nun hat sich seit längerem im Rahmen von Stadt- und Gästeführungen ein Interesse an Begegnungen mit sozial-historischen Stationen der Armut, der Kriegswirren, aber auch der Kriminalität und vulgär-lasziver Sittenlosigkeit im eigenen Land entwickelt.

Etabliert sind Besichtigungen von Burgverliesen, Kerkern und Folterkammern in mittelalterlichen Städten, Führungen durch ehemalige Moorkolonien mit ihren unmenschlichen Arbeits- und Lebensbedingungen sowie Besuche von Bunkermuseen aus der Zeit des Zweiten Weltkriegs.

Seit einigen Jahren erfolgen auch Stadtführungen in die sozial schwächeren, von Migration multikulturell geprägten Stadtteile, beispielsweise in Berlin (Der Beauftrage des Senats für Integration und Migration 2011), in denen es Spannungen und Konflikte gibt, die aber auch sozialkulturelle Kreativität freisetzen. 
Dadurch sollen bei den Teilnehmern rigide »mental maps « verändert werden und stigmatisierte Wohnquartiere und ihre Bewohner, ähnlich wie dies mit den Slum-Touren beabsichtigt ist (Burgold u. a. 2013, 102), aufgewertet werden. Regen Zuspruch verzeichnen zudem in jüngster Zeit deutschlandweit Begehungen von Gefängnissen an »Tagen der offenen Tür « als eine neue Form von Public Viewing (vgl. Westfälische Nachrichten 15.09.2013). Zu buchen sind ferner schaurig-schöne Events mit »Hexen, Huren und Henkersknechten« - lukullisch garniert mit einem obligatorischen »Krimi-Dinner«. Aber es werden auch Stadtführungen offeriert, die weniger der sozialen Erinnerungs- und Verantwortungskultur dienen, sondern die mentalitätstypisch die Klischees von "Hamburgs sündiger Meile « präsentieren - zunehmend gendergerecht als »Mädels-Tour « zu buchen, unter dem Motto: "St. Pauli Haut nah".

Auch hier gilt es bei der Wahrnehmung und Gestaltung sozial-geschichtlicher Begegnungen sowie zeitgenössischer touristischer Einblicke in soziale Randzonen bürgerlicher Normalität und Sittlichkeit einschließlich ihrer doppelter Moral -, durch sachgerechte Information und der Anbahnung mitmenschlicher Empathie, vorzubeugen, dass nicht unangemessene Verallgemeinerungen, Vorurteile und Stigmatisierungen verstärkt werden auf der Grundlage einer »falschen Theorie von der Richtigkeit des Ersteindrucks«.

Immer ist im Zusammenhang mit der "Sozialen Frage« die Menschenwürde zu wahren und darauf hinzuwirken, dass die gebotenen Attraktionen und Shows auch als solche wahrgenommen werden und dass marginalisierende Lebensbedingungen nicht unhinterfragt als alltäglich Realität eines einfachen, im Grunde aber doch zufriedenstellenden Lebens fehlinterpretiert werden. Es ist deshalb geboten, aufscheinende problematische Bedingungen (von Migration, Arbeitsvermittlungshemmnissen, Zwangsprostitution und Deliquenz) nicht zu bagatellisieren, sondern angemessen zu thematisieren.

\section{Konsumkritische Stadtführungen: "Global denken - lokal handeln"}

Eine neuere Entwicklung im Rahmen von Stadtführungen in Deutschland beabsichtigt, aktuelle Einblicke in globale, also weltweite soziale Problemlagen zu vermitteln. Im Sinne von »global denken und lokal handeln ", soll durch eine "glokale « Perspektive ein konsumreflexives Verhalten gefördert werden.

So hat sich beispielsweise bundesweit in annähernd 50 Städten der sogenannte »Weltbewusst Stadtrundgang « etabliert. Es handelt sich dabei um Stadtführungen, die als eine »Entdeckungsreise durch die globalisierte Marken- und Produktwelt « deutscher Innenstädte konzipiert sind. Vermittelt werden $\mathrm{Zu}$ sammenhänge zwischen individuellem Konsumverhalten auf der lokalen Ebene und den globalisierten ökosozialen Missständen in anderen Regionen der Welt.

Informiert wird bei solchem »Sightseeing im Konsumdschungel« darüber, wie durch bewussten, umwelt-, sozial- und nachhaltigkeitsverträglichen Konsum eine positive Einflussnahme auf die weltweite "Soziale Frage « und dadurch ein Mehr an jedoch aus unterschiedlichen Gründen ein Interesse an vertieften Informationen über die Ursachen spezifischer Notlagen, die bestehenden Hilfeangebote sowie die Nachhaltigkeit sozialer Hilfeleistungen im Sinne des »Social Return on Investment .

Als Anbieter solcher Unternehmungen sind in der Regel vereinsmäßig organisierte örtliche Sozialakteure aktiv. Mit ihren »Touren zu den Stationen der Armut « wollen sie in einer sozial-ethischen Perspektive authentisch informieren über lokale Sozialleistungs-, Hilfe- und Unterstützungsangebote und zwar auf dem Wege unmittelbarer Begehung und Inaugenscheinnahme.

Dabei versteht es sich von selbst, dass mit diesen Touren nicht etwa die bigotte Entrüstung über obdachlose Menschen und andere Personen mit Hilfe- und Unterstützungsbedarf bedient werden soll.

\section{"Mit Slum-Touren und Städteführungen sollen auch bürgerschaftliches Engagement gefördert werden"}

globaler sozialer Gerechtigkeit möglich wird. Als erfolgreiche Maßnahme für solcherart ressourcengerechtere Weltgestaltung kann auf die Unterstützung der Initiativen für »fairen Handel « verwiesen werden, wie sie etwa durch (Dritte-) Welt-Läden erfolgt, oder durch den Kauf von kinderarbeitsfreien Produkten (www. weltbewusst.org/stadtrundgang).

\section{Pro-soziale Stadtführungen zur Förderung einer "Kultur der Solidarität»}

Eine weitere neuere Entwicklung, deren Intention hier des Näheren vorgestellt werden soll, stellen Exkursionen dar, die Einblicke in lokale soziale Brennpunkte vermitteln wollen.

Zielgruppe für eine solche »Tour zu den Stationen der Armut « in der heimatlichen Kommune sind dabei nicht primär auswärtige Touristen, sondern Einheimische, die sich mit der "Sozialen Frage im unmittelbaren Lebenszusammenhang ihrer Kommune beschäftigen wollen. Sie sind in der Regel zwar durch die lokalen Medien über diverse soziale Hilfebedarfe von Menschen in schwierigen Lebenslagen und entsprechende Unterstützungsangebote in ihrer Stadt informiert, haben
Vielmehr geht es, in Weiterführung des vorstehenden Beispiels, um Informationen über Ursachen und Auswirkungen von Obdachlosigkeit sowie über vorhandene Hilfestrukturen vor Ort für Personen mit geringen materiellen, kulturellen und sozialen Ressourcen. Gleichwohl ist mit diesen Touren nicht eine unmittelbar sozialcaritativ unterstützende Begegnung mit Personen in prekären Lebensumständen intendiert, noch gar eine Kontrolle ihrer Würdigkeit zum Empfang privater Wohltätigkeit, wie sie etwa im 19. Jahrhundert in England durch sogenannte »friendly visitors « betrieben wurde (Wendt 1990, 136, 132).

Wie in immer mehr Städten Deutschlands, so gibt es auch in Hannover einen "Sozialen Stadtrundgang «. Er wird von der gemeinnützigen sozialen Straßenzeitungsinitiative "Asphalt-Magazin « veranstaltet, bei dem Verkäufer des Straßenmagazins die Führung übernehmen und diejenigen »interessanten Punkte Hannovers ansteuern, wo Menschen trotz leerem Geldbeutel guten Rat und günstige Unterstützung erhalten. Sei es die kompetente Umzugshilfe, ein warmes Mittagessen, im Winter wie im Sommer passende Kleidung für Groß und Klein, Beratung und Trost nach Gewalterfahrung oder auch die 
Möglichkeit, selbst Asphalt-VerkäuferIn zu werden" (Busse 2014).

Einen guten Überblick über Stationen einer Sozialen Stadtführung bietet Göttingen, dort stehen folgende Einrichtungen auf dem Programm: die Göttingen-Tafel, das Projekt Blechtrommel der Jugendhilfe Göttingen, das Männerwohnheim der Heilsarmee, der Verein Kore, das Migrationszentrum für Stadt und Landkreis, die sozialpsychiatrische Beratungsstelle Shelter, das Drogenberatungszentrum für illegale Drogen des Diakonieverbandes, der Mittagstisch für Obdachlose und Arme der katholischen St.-Michael-Gemeinde, die Arbeitswohlfahrt mit ihrer Schuldnerberatung, der Jobclub 50+, das Jobcenter Jugend, das städtische Jugendprojekt Kontur, die Ambulante Hilfe (ehemals Wohnungsnothilfe), die Straßensozialarbeit des Diakonieverbandes Göttingen, die therapeutische Jugendhilfeeinrichtung Wohnung und Betreuung des Sozialpädagogischen Schüler- und Lehrlingszentrum, die Bahnhofsmission und das Göttinger soziale Straßenmagazin TagesSatz (Göttinger Tageblatt 2009). Ein exemplarisches Bild über Soziale Stadtführungen bietet zudem die Video-Dokumentation des "Sozialen Stadtrundgangs in Basel der Schweizer Vereinigung Surprise, die das gleichnamige Straßenmagazin vertreibt (Surprise 2013).

Indem den Teilnehmern dieser Sozialexkursionen die Möglichkeit eröffnet wird, sich über bestehende Hilfebedarfe und Unterstützungsangebote vor Ort zu informieren, können sie ein realistischeres Bild über die strukturellen Bedingungen, Ziele und mögliche Erfolge sozialer Arbeit gewinnen (vgl. Gangway 2012).

Dadurch werden möglicherweise bestehende generalisierte Vorurteile über die Irrelevanz bestehender sozialer Hilfen minimiert und die oftmals vorhandene Tendenz zu undifferenzierten individuellen Schuldzuweisungen kann hinterfragt werden. Vor allem aber soll durch kritisch aufklärende und differenzierende soziale Informationsbemühungen (vgl. Geiling 2005) die Sinnhaftigkeit einer prosozial und aktiv gelebten $»$ Kultur der Solidarität« verdeutlicht werden.

Dadurch besteht die Möglichkeit für sozialstaatliches, wohlfahrtsverbandliches und - durchaus auf die Teilnehmer bezogenes - bürgerschaftliches Engagement zu sensibilisieren, das zu Auswegen aus Armut und sozialen Notlagen beitragen möchte (Wilken 2010,31), indem auf
Förderung der Eigenaktivität gesetzt wird, die erlernte Hilflosigkeit und inszenierte Hilfsbedürftigkeit überwinden will.

Im Vergleich mit dem Slum-Tourismus in den touristischen Destinationen weltweit geht es bei den geführten heimischen Sozialtouren zu den Stationen der Armut durchaus auch um Verständnis für die Welt der betroffenen Menschen, aber es geht nicht so sehr um eine direkte Begegnung mit ihnen in ihren sozial und kulturell randständigen Milieus und um die unmittelbare Wahrnehmung der dort herrschenden problematischen Lebensverhältnissen. Erst recht geht es nicht um eine voyeuristische Zurschaustellung von armen, verwahrlosten, alkohol- und drogenabhängigen Menschen in prekären Lebenslagen.

Vielmehr ist die Intention dieser geführten Einblicke in lokale soziale Brennpunkte und die bestehenden Unterstützungsangebote auf eine nachhaltige Stärkung des bürgerschaftlichsozialen Verantwortungsbewusstseins in der heimischen Kommune gerichtet, das in der Lage sein sollte, angesichts exkludierender und benachteiligender Bedingungen, die Selbstevidenz des Sozialen im Kontinuum von Selbstsorge, Mitsorge und Fürsorge zu sichern und im Blicke auf soziale Inklusion und Teilhabe zukunftsgerecht und sozialverträglich zu befördern (ebd. 36).

\section{Literatur}

Burgold, J., Frenzel, F., Rolfes, M.: Observations on slums and their touristfication. In: DIE ERDE. Journal of the Geographical Society of Berlin. Vol. 144, No. 2/2013, 99-104

Busse, S.: Asphalts Soziale Stadtführung - gut angenommen. In: www.asphalt-magazin.de/ de/42568-Sozialer_Stadtrundgang (Zugriff 01.08.2014).

Der Beauftragte des Senats für Integration und Migration (Hg.): Stadt ist Migration. Die Berliner Route der Migration. Grundlagen, Kommentare, Skizzen. Berlin 2011.

\section{Frenzel, F., Koens, K., Steinbrink, M. (Hg.):}

Slum tourism. Poverty, power and ethiks. Routledge, New York 2012.

Frenzel, F.: Slum tourism in the context of the tourism and poverty (relief) debate. In: DIE ERDE. Journal of the Geographical Society of Berlin. Vol. 144, No. 2/2013, 117-128.

Gangway, e.V. - Straßensozialarbeit in Berlin: Straßenzeitung, Stadtführungen, Obdachlosenuni \& Co. Wohnungslose gemeinsam aktiv! Sammlung von Best-Practice-Beispielen. Re- cherche im Auftrag von Gangway e.V. - Erstellt von Maik Eimertenbrink, Berlin 2012: www.armutsnetzwerk.de/data/Recherche_WohnungsloseGemeinsamAktiv.pdf (Zugriff 04.08.2014).

Geiling, H.: Zur Theorie und Methode einer Stadtteilanalyse. 2005: www.ipw. uni-hannover.de/index.php?elD=tx_ nawsecured $\&$ \&u=o\&file=uploads $/$ media $/$ Stadt teilanalyseoo1.pdf\&t=1406990201\&hash $=6$ e3 1c4c61ca65b23a8eag629e6dbobe696d4f6bo (Zugriff 20.08.2014).

Göttinger Tageblatt: Soziale Einrichtungen als Sehenswürdigkeit. 29.11.2009: www.goettinger-tageblatt.de/Nachrichten/Goettingen/ Uebersicht/Soziale-Einrichtungen-als-Sehenswuerdigkeit (Zugriff 01.08.2014).

Gutekunst, M.: Nächster Halt: Armenviertel. Die ganz normalen Leute, wie du und ich. In: fluter. Magazin der Bundeszentrale für politische Bildung (Themen-Archiv), 28.1.2013. www.fluter.de/de/118/thema/11250/ (Zugriff 18.07.2014).

Loiperdinger, M. und Vogl-Bienek, L.: Im Zeichen der Armut. Zwischen medialer Inszenierung und sozialem Abbild. In: forschung - Das Magazin der Deutschen Forschungsgemeinschaft. DFG, Presse- und Öffentlichkeitsarbeit, Bonn 2/2013, 10-14

Rolfes, M., Steinbrink, M., Uhl, C.: Townships as Attraction. An Empirical Study of Township tourism in Cape Town. Universitätsverlag (Praxis Kultur- und Sozialgeographie - PKS 46) Potsdam 2009

"Screening the Poor 1888-1914". DVD-Edition,

2. Auflage 2011: www.edition-filmmuseum. com/product_info.php/info/p130_Screeningthe-Poor-1888-1914.html.

Surprise 2013. "Soziale Stadtrund-

gang" in Basel: www.youtube.com/ watch? $v=q D w p M m h r S m Y$ (Zugriff 02.08.2014).

TourismWatch - Informationsdienst Tourismus und Entwicklung Nr. 75. Herausgeber: Brot für die Welt - Evangelischer Entwicklungsdienst, Tourism Watch, Berlin, Juni 2014a, 6-7.

TourismWatch - Informationsdienst Tourismus und Entwicklung: Fair Reisen mit Herz und Verstand. Herausgeber: Brot für die Welt Evangelischer Entwicklungsdienst, Tourism Wacht, Berlin 2014b.

Wendt, W. R.: Geschichte der sozialen Arbeit. Stuttgart 1990.

Westfälische Nachrichten. Leben hinter Gittern. Tag der offenen Tür: Tausende besuchten die JVA Münster. 15.09.2013.

Wilken, U.: Der Verlust der Selbstevidenz des Sozialen als ethische Herausforderung. Essay über Perspektiven für eine erneuerte Kultur der Solidarität. In: Wilken, U./Thole, W. (Hg.): Kulturen Soziale Arbeit. Profession und Disziplin im gesellschaftlichen Wandel. Wiesbaden 2010, 27-37. 
"Ein Land mit nur einer Sprache und einer Sitte ist schwach und gebrechlich. Daher ehre die Fremden und hol sie ins Land."

Stephan I., erster König von Ungarn (969-1038)

"Eigenartig / Wie das Wort eigenartig / Es als fremdartig hinstellt / Eine eigene Art zu haben." Erich Fried, österreichischer Schriftsteller (1921-1988)

„Um fremden Wert willig und frei anzuerkennen,

muss man eigenen haben. "

Arthur Schopenhauer, deutscher Philosoph (1788-1860)

"Am Chauvinismus ist nicht so sehr die Abgrenzung gegen die fremden Nationen als die Liebe zu eigenen unsympathisch." Karl Kraus, österreichischer Schriftsteller (1874-1936)

"Fremde, die vor einer Weile die Gegend besiedelt haben, sind der Meinung, es sollten keine Fremden in der Gegend siedeln. " Jürgen Becker, deutscher Schriftsteller (geb. 1932)

„) Fremd ist der Fremde nur in der Fremde. " Karl Valentin, deutscher Kabarettist (1882-1948)

„Die Germanen selbst sind Ureinwohner, möchte ich meinen, und von Zuwanderung und gastlicher Aufnahme fremder Völker gänzlich unberührt. (...) Wer hätte auch - abgesehen von den Gefahren des schrecklichen und unbekannten Meeres - Asien oder Afrika oder Italien verlassen und Germanien aufsuchen sollen, landschaftlich ohne Reiz, rau im Klima, trostlos für den Bebauer wie den Beschauer, es müsse denn seine Heimat sein? "

Publius Cornelius Tacitus, Germania (römischer Geschichtsschreiber, um 55-115) 